\title{
A CURVATURE NORMAL FORM FOR 4-DIMENSIONAL KÄHLER MANIFOLDS
}

\author{
DAVID L. JOHNSON
}

\begin{abstract}
A curvature operator $R$ is said to possess a normal form relative to some space of curvature operators $\mathscr{P}$ if $R$ is determined uniquely in $\mathcal{P}$ by the critical points and critical values of the associated sectional curvature function. It is shown

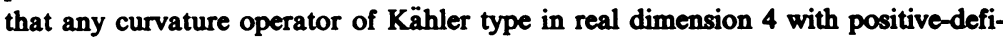
nite Ricci curvature has a normal form relative to the space of all Kähler operators.
\end{abstract}

In [2] the author showed that a generic set of curvature operators of Kähler type with positive-definite Ricci tensor in real dimensions 4 and 6 possess normal forms, analogous to the diagonalization of symmetric operators but related to the sectional curvature. This concept arose out of work of Singer and Thorpe [3]; their normal form for Einstein 4-manifolds simplified greatly many classical integral expressions and showed plainly the relationship between curvature properties and characteristic numbers for these manifolds.

In this note, the author hopes to rectify, in real dimension 4, the major drawbacks of the normal form theorem proven in [2], which were the implicit nature of the normal form itself and the involved topological methods used in its proof.

The author wishes to express his gratitude to the Departamento de Geometria y Topologia, Facultad de Ciencias Matemáticas, Universidad de Valencia, for support provided during the preparation of this note. I am especially grateful to Professors A. M. Naveira and A. Ferrandez for many enlightening discussions during my stay.

1. Definitions and statement of the theorem. A curvature operator $R \in R(V)$ on a finite-dimensional inner product space $V$ is a symmetric operator on $\Lambda^{2}(V)$. $R$ is called proper if it satisfies the standard first Bianchi identity. If $V$ is a hermitian complex vector space with complex structure automorphism $J: V \rightarrow V$, define $J \in \Re(V)$ by $J(v \wedge w)=J v \wedge J w . R \in R(V)$ is called Kähler, denoted by $R \in \mathcal{K}(V)$, if $R J=J R=R$.

The Grassmannian $G(2, V)$ of oriented 2-planes in $V$ is identified with $\{\xi \in$ $\left.\Lambda^{2}(V)|| \xi \mid=1, \xi \wedge \xi=0\right\}$ [3]. The sectional curvature $r_{R}: G(2, V) \rightarrow \mathbf{R}$ of $R \in$ $\Re(V)$ is then defined by $r_{R}(P)=\langle R P, P\rangle$.

Let $\mathscr{P} \subset \mathcal{R}(V)$ be a subspace. A proper $R \in \mathcal{P}$ has a normal form relative to $\mathscr{P}$ if there is a set $\left\{P_{i}\right\}$ of critical points of the sectional curvature $r_{R}$ so that, if

Received by the editors July 30, 1979.

AMS (MOS) subject classifications (1970). Primary 53B20, 53B35; Secondary 53A55, 53C25.

Key words and phrases. Sectional curvature, algebraic curvature tensor, Kähler curvture operator. 
$R^{\prime} \in \mathcal{P}$ is proper and $r_{R^{\prime}}$ has critical points at $\left\{P_{i}\right\}$ with $r_{R^{\prime}}\left(P_{i}\right)=r_{R}\left(P_{i}\right)$, then $R^{\prime}=R$.

THEOREM 1.1. Let $V \simeq \mathbf{C}^{2}$. Then each proper $R \in \mathcal{K}(V)$ with positive-definite Ricci tensor has a normal form relative to $\mathcal{K}(V)$.

2. Properties of $\mathcal{K}(V)$. Making the standard identification of $o(V) \simeq \Lambda^{2}(V)$, the subspace $u(V) \subset o(V)$ of skew-hermitian operators is the set of all $J$-invariant elements of $\Lambda^{2}(V)$. Define $I \in u(V)$ by identifying the operator $J$ with an element $I$ of $\Lambda^{2}(V)$. In a unitary basis $\left\{v_{1}, v_{1^{*}}=J v_{1}, \ldots, v_{n}, v_{n^{*}}\right\}, I=\Sigma_{i} v_{i} \wedge v_{i^{*}}$

In [2] the following useful formulas are derived; their proofs are simple calculations.

Proposition 2.1. Let $\rho(R): V \rightarrow V$ be the Ricci tensor of $R$. Then if $R$ is proper

(1) $\langle\rho(R) v, w\rangle=\langle R(I), v \wedge J w\rangle$;

(2) $\rho(R)$ is positive-definite if and only if $\langle R(I), v \wedge J v\rangle>0$ for each $v \in V$.

A plane $P \in G(2, V)$ will be called holomorphic if $J P=P$. The set of all holomorphic planes is clearly 2 copies ( + and - ) of $\mathbf{C P}(V)$.

Proposition 2.2. (1) $P \in \pm \mathbf{C P}(V)$ is $\mid a_{\mid}$critical point of $r_{R}$ if and only if $P$ is a critical point of $r_{R \mid \pm C P(V)}$. In the case $V \simeq C^{2}, P$ is a holomorphic critical point of $r_{R}$ if and only if $R P=A P+B^{\prime} * P$, where $A=r_{R}(P)$ and $*$ is the Hodge star operator.

(2) Let $V \simeq \mathbf{C}^{2} . Q \in G(2, V)-( \pm \mathbf{C P}(V))$ is a critical point of $r_{R}$ if and only if $R Q=A(Q+J Q-\langle Q, I\rangle I)$, where $A=r_{R}(Q)$. Thus $\langle R Q, I\rangle=0$.

COROllary 2.3. For $V \simeq C^{2}$, there are always at least 2 holomorphic critical points. If $\rho(R)$ is positive-definite, there are at least 3 nonholomorphic critical points $\left\{Q_{i}\right\}$ with $\left\{Q_{i}+J Q_{i}\right\}$ linearly independent.

Proof. The first statement is clear. For the second, note that each nonholomorphic critical point $Q$ satisfies

$$
Q+J Q \in\{\xi \in u(V) \mid\langle R I, \xi\rangle=0\}=R I^{\perp} \text {. }
$$

Also, each $\xi \in R I^{\perp}$, up to a scalar factor, is of the form $Q+J Q$ for some plane $Q \in G(2, V)-( \pm \mathrm{CP}(V))$, since $\xi=a v_{1} \wedge v_{1^{*}}+b v_{2} \wedge v_{2^{*}}$ for an appropriate choice of unitary basis. $\rho(R)$ positive-definite implies that $a b<0$. Choose a basis so that $a>0, b<0$. Then, if $Q=\left(\sqrt{a} v_{1}+\sqrt{-b} v_{2^{*}}\right) \wedge\left(\sqrt{a} v_{1^{*}}+\sqrt{-b} v_{2}\right)$, $Q+J Q=2 \xi$. Thus $R I^{\perp}$ is in essence the space of possible nonholomorphic critical points of $R$. Let $A: R I^{\perp} \rightarrow I^{\perp}(=\{\xi \in u(V) \mid\langle\xi, I\rangle=0\})$ be the orthogonal projection, $A(\xi)=\xi-\frac{1}{2}\langle\xi, I\rangle I$. As $\langle R I, I\rangle>0, A$ is an isomorphism.

The operator $R A^{-1}: I^{\perp} \rightarrow I^{\perp}$ is symmetric, since, for $\alpha, \beta \in R I^{\perp},\left\langle R A^{-1}(A \alpha)\right.$, $A \beta\rangle=\langle R \alpha, A \beta\rangle=\langle R \alpha, \beta\rangle=\langle\alpha, R \beta\rangle=\left\langle A \alpha, R A^{-1}(A \beta)\right\rangle$. Clearly any eigenvector $\xi$ of $R A^{-1}$ is of the form $\xi=\lambda A(Q+J Q)$, where $Q$ is a critical plane of $r_{R}$. As $\operatorname{dim}\left(I^{\perp}\right)=3$ the corollary is finished.

3. Proof of Theorem 1.1. Let $R$ be as in the statement of the theorem, with critical planes $\left\{P_{1}, P_{2}, Q_{1}, Q_{2}, Q_{3}\right\}$ as given in Corollary 2.3. If $R^{\prime}$ is another 
operator with the same critical points and values, $R^{\prime} I^{\perp}=R I^{\perp}$; moreover, $R-R^{\prime}$ $=T$ is zero on $R I^{\perp}$, as $\left(R-R^{\prime}\right) A^{-1}=0$ ( $A$ is the same for both operators). Thus, $T(\xi)=\mu\langle\xi, R I>R I$ as $T$ is a symmetric operator on $u(V)$ with $R I$ as the only possible nonzero eigenvector. However, $0=\left\langle T P_{1}, P_{1}\right\rangle=\mu\left\langle P_{1}, R I\right\rangle^{2}$ with $\left\langle R I, P_{1}\right\rangle>0$, thus $T=0$.

REMARK. It is possible to extend this result to all proper $R \in \mathcal{K}(V)$, but the proof is extremely inelegant [1].

4. An application. It is not difficult to apply the general techniques of this analysis, though not necessarily the normal form itself, to establish the classical Hopf conjecture in dimension 4 for Kähler manifolds, that nonnegative curvature implies nonnegative Euler characteristic. Indeed, one need only repeat Milnor's well-known proof in the general dimension 4 case, applied to any one critical point. Alternately, it is straightforward to show that the algebraic properties of operators with positive curvature are quite restricted.

Proposition 4.1. If $V \simeq \mathrm{C}^{2}$, any proper $R \in K(V)$ with nonnegative sectional curvature is positive semidefinite.

Proof. If $R$ is not positive semidefinite, let $\xi$ be an eigenvector with negative eigenvalue. $\xi$ must lie in $u(V)$, so for an appropriate choice of unitary basis, $\xi=a v_{1} \wedge v_{1^{*}}+b v_{2} \wedge v_{2 *}$. As $r_{R}>0, a b<0$. As before, choose the basis so that $a>0, b<0$. Then $Q=\left(\sqrt{a} v_{1}+\sqrt{-b} v_{2^{*}}\right) \wedge\left(\sqrt{a} v_{1^{*}}+\sqrt{-b} v_{2}\right)$ will satisfy $Q+J Q=2 \xi$ and $r_{R}(Q)=\langle R Q, Q\rangle=\langle R \xi, \xi\rangle\langle 0$, contradicting the fact that $r_{R}>0$.

An unpublished but well-known result of B. Kostant states that, if $R$ is any proper, positive semidefinite curvature operator, then the Gauss-Bonnet integrand is nonnegative. Thus,

COROLlARY 4.2. If $M$ is a 4 real-dimensional Kähler manifold with nonnegative curvature, the Euler characteristic of $M$ is also nonnegtive.

REMARK. In a forthcoming article, Curvature and Euler characteristic for six-dimensional Kähler manifolds, the author is able to verify this conjecture for six-dimensional Kähler manifolds as well, using techniques developed in [2].

\section{REFERENCES}

1. D. L. Johnson, A normal form for curvature, Ph.D. Thesis, M.I.T., 1977.

2. Sectional curvature and curvature normal forms, Michigan Math. J. (to appear).

3. I. M. Singer and J. A. Thorpe, The curvature of 4-dimensional Einstein space, Global Analysis, Univ. of Tokyo Press, Tokyo, 1969, pp. 355-365.

Department of Mathematics, Texas A \& University, College Station, Texas 77843

Current address: Department of Mathematics, Rice University, Houston, Texas 77001 